\title{
USING REMOTE SENSING DATA TO CREATE MAPS OF VEGETATION AND RELIEF FOR NATURAL RESOURCE MANAGEMENT OF A LARGE ADMINISTRATIVE REGION
}

\author{
O. V. Artemeva ${ }^{1, *}$, S. Zareie ${ }^{2}$, Y. Elhaei ${ }^{2}$, N. A. Pozdnyakova ${ }^{1}$, N. D. Vasilev ${ }^{1}$ \\ ${ }^{1}$ St. Petersburg State University Institute of Earth Science, St. Petersburg, Russia - o.artemieva@spbu.ru, qlnat@mail.ru, \\ vasilevnikolay18@gmail.com \\ ${ }^{2}$ Department of Remote Sensing and GIS, Faculty of Earth Sciences, Shahid Chamran University of Ahvaz, Ahvaz, Iran - \\ s.zareie2018@gmail.com, yasaminelh@gmail.com
}

KEY WORDS: Remote sensing data, Vegetation maps, Relief maps, Data classification, Administrative region

\begin{abstract}
:
The authors offer methods for mapping nature, in particular, vegetation and relief maps using remote sensing data. These thematic maps are most often used by administrators of different levels for environmental and territorial management. In the Russian Federation administrative territories occupied large areas. The algorithm for constructing visual models using remote sensing data for large administrative areas differs from the algorithms for working with small territories. Automated mapping method includes the analysis of the territory by indicators of topography and dominant vegetation, the selection of satellite images, processing, composing mosaics, composites, classification of plant objects, post-processing. The authors offer to use a specific data source, because the quality of the materials is sufficient for working with large areas. Classifications - the most complicated section. At the moment, scientists have not proposed an unambiguous solution to the choice of algorithm. However, the authors of this study experimentally came to the most convenient algorithm, which we characterize as the main one precisely for the purposes of managing natural resources of large administrative structures (regions with legally fixed boundaries). Examples of the thematic maps fragments and results of intermediate versions of visual models built by automated methods are given. The potential use of methods by municipal employees, rather than narrow specialists, was taken into account. In this regard, the value of the study is an exclusively applied nature and can be used in the administrative structures of the executive authorities
\end{abstract}

\section{INTRODUCTION}

The natural resources management requires a visual representation of the administrative territory. An evaluation of forests, agricultural land and different levels biocenosis based on remote sensing and the compilation of a nature maps - is a priority. The most convenient and informative visual models are the maps of nature. The compiling of socio-economic maps may be based on statistical data. But the compiling of nature maps requires not only the use of statistics, but also a quite complex methodological algorithm for processing remote sensing data.

Heads of municipalities, regional, city administrators and other top managers have long realized that without automated cartographic methods, it is difficult to have progress in the economy and in the process of managing natural resources. A modern automated methods of mapping based on remote sensing data processing. Top managers of the regional administrations ask for scientific organizations and higher education institutions to cooperate. This is where specialists in the field of remote sensing data processing work. Moreover, there is already a tendency to invite to work even in the executive authorities and regional governments specialists working with coordinate information.

Natural Resource Management is based on a multidimensional approach to the components of nature. When a legally established region becomes larger - the harder it is to explore the human pressure. And the more difficult it is to make decisions on the management of the territory. Russian Federation - a country in which administrative regions may occupy huge areas. Due to this fact it is very difficult to put together the original data, and, moreover, to apply the standard of remote sensing data processing algorithms.

Remote sensing data is currently the basis for compiling maps of nature. The most difficult is to work with satellite data for compiling maps of vegetation. The task of interpreting the classifications of the landscape, plant communities, urbanized areas and agricultural land for administrative region management - one of the most urgent. In addition, plant communities are naturally dependent on topography. Therefore, first of all, relief maps should be created, and then maps of plant communities. In the Russian Federation, the use of relief maps is extremely important for agriculture. The country has seen increased growth of ravines, reducing arable land and contributing to the washing away of valuable soils. Moreover, Russian agriculture is a large-scale mechanized economy. It is necessary to consider the relief, as the performance of machines may not be high enough. Therefore, the compiling of relief maps along with vegetation maps based on remote sensing data are very important now for science, industry, and agriculture of individual regions.

We offer several methods for creating thematic maps of nature, this could be used by administrators of large territorial

\footnotetext{
* Corresponding author
} 
entities. These maps are the basis for spatial planning.

\section{PROPOSED METHODS}

The map compilation of nature process has a number of features. The basic principles: conservation of geometric image and natural pattern; using geographical and indicator localization, as well as geographical interpolation; matching different maps one to each other. (Bozilina, et al., 2016), (Sochava, 1979) (Zaruzkaja, et al., 1989) Relief is the basis and foundation of the entire geographical pattern. Therefore, before compiling vegetation maps, it is necessary to compile relief maps.

\subsection{Compilation the maps of relief.}

The task of compiling a relief maps based on remote sensing data has been solved by many authors. (Seger, M, 1989), (Metelka, V., et al.)

The first task is data acquisition. There are several sources of open source data where we can find the right tiles (SRTM): United States Geological Survey [Earthexplorer.usgs.gov, 2018a, b] or Shuttle Radar Topography Mission [Srtm.csi.cgiar .org / srtmdata, 2018] and others. We have tried to use other data, but these were inconvenient to use in our context and often incorrect.

The second task is the construction of isolines (isohyps). The distance between them must be chosen taking into account the peculiarities of the territory and "with excess". For example, if the topography is flat, then it makes sense to choose a smaller section. If the scale has been uneven, then chose the smallest of the sections; during the further analysis, it is possible to remove excess isohyps.

The next stage is the creation of relief shading. The relief analysis tool "Shadow Relief" was used. With the help of this tool was made shadow relief, representing a bitmap, which contains information on the distribution of sunlight on the territory. (Kennelly, 2008) It is possible to adjust the direction of the light source ("Sun") and its height above the horizon. If the absolute elevations are insignificant or there is relatively flat terrain, then it is permissible to set a vertical scale greater than "one". The resulting shading will correspond to the relief forms exceeding the real picture a specified number of times.

The fourth stage is the post-processing of the shading. After the construction of the relief shading it is necessary to clarify what values it takes. If the majority is below $150-190$, it is necessary to bring the raster to a new range of values in such a way that the spread of values is no more than 100 and the whole range lies as close as possible to 255 . To this end, we offer the following steps:

$$
\text { Shadow_raster } * \mathrm{k}+\mathrm{q} \text {, }
$$

Shadow_raster - shading, $\mathrm{k}$ - the scale factor, the approximate value of which can be taken equal to:

$$
\mathrm{k}=100 / \max \mathbb{\mathrm { I }}-\mathrm{min} \rrbracket \text {, }
$$

max/min - maximum / minimum value raster Shadow_raster; $q$ - a shift whose value is equal to:

$$
q=\lfloor 254-k * \max \rfloor,
$$

Operator \」means rounding from the bottom. (Figure 1)

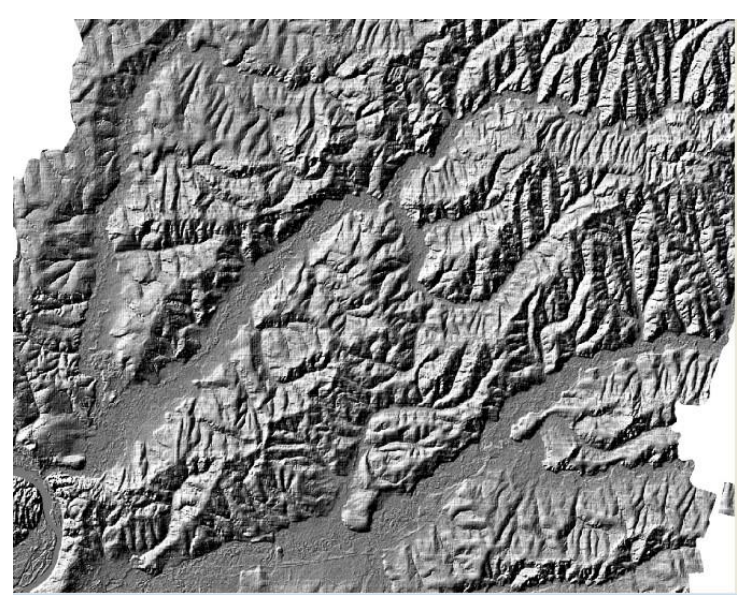

Figure 1. The results of the relief shading construction

The fifth stage - the preparation of a color scale and the creation of a color relief. For the next step, we will need to create a file with the txt extension of the following format:

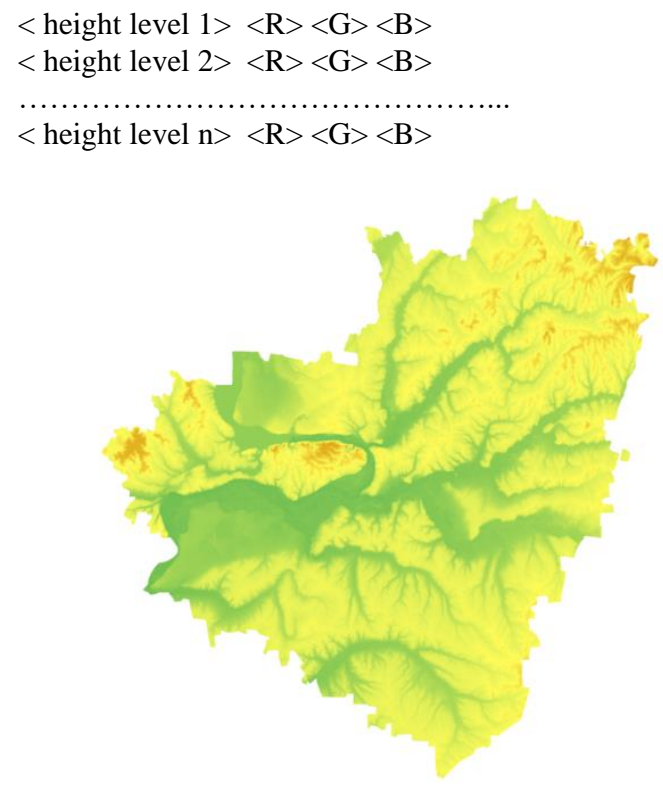

Figure 2. The results of the colorized relief construction.

Here $\langle$ height level $\mathrm{k}\rangle$ is the height of the level, $\langle\mathrm{R}\rangle$ is the red component of the color, $\langle\mathrm{G}\rangle$ is the green component of the color, $\langle\mathrm{B}\rangle$ is the blue component of the color (the color in which the current level will be painted [k: $\mathrm{k}+1])$.

High-altitude steps need to be built only after working with contours, because they determine their number and borders.

The next stage - the creation of colorized relief. It is better to use the tool "Color relief". The basis of this operation - the creation of layered coloring. Unlike traditional options, where a discrete representation of the color scale is possible, in this algorithm it will represent a continuous (linear scale). (Figure 2.)

The final stage is the creation of the final image. We offer two options. 
Option 1: merge relief with shading and color relief. (Figure 3) The essence of this method is that the original colorized relief is converted from the RGB color system to HSV. Thereafter, the value of $\mathrm{H}$ is replaced by a value $\mathrm{H}$ relief shading. And then converted to RGB again. This approach allows you to get the color relief in the same tones, but darker depending on the light. During the post-processing of shading should be careful not to get too dark.

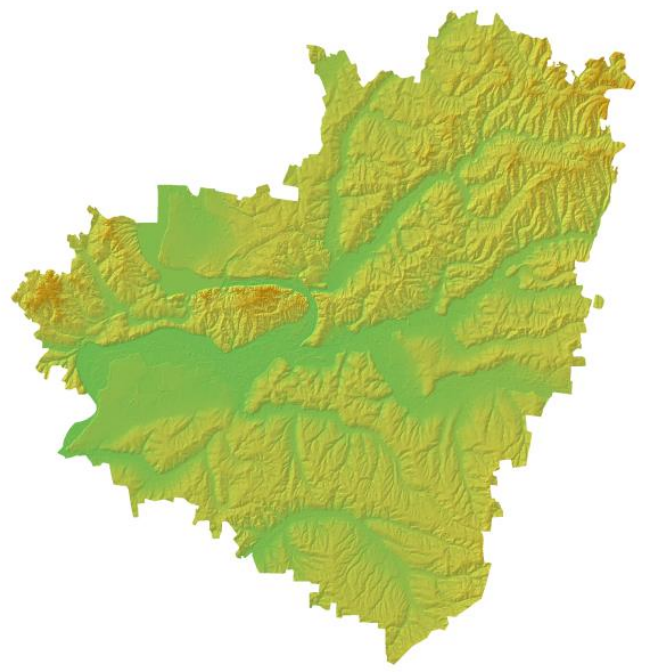

Figure 3. Merge relief with shading and color relief

Option 2: overlay layers with transparency setting. A color relief serves as a substrate, on which a layer of shading is superimposed. The latter can be set up any transparency. (Figure 4)

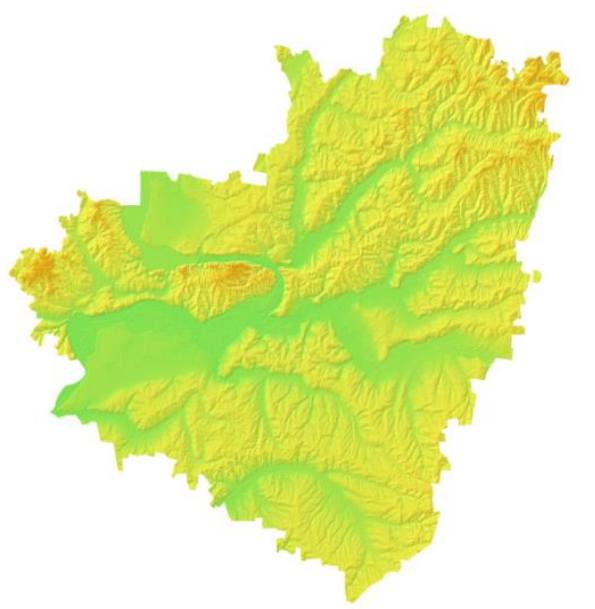

Figure 4. Overlay layers with transparency setting

The most spectacular is the first method. The simplest is the second one. (Figure 5,6)

\subsection{Compilation the maps of vegetation.}

Currently, there are several satellite systems with the possibility of their use of satellite images for practical purposes. However, we believe that only the Landsat-8 (or Sentinel-2) satellite

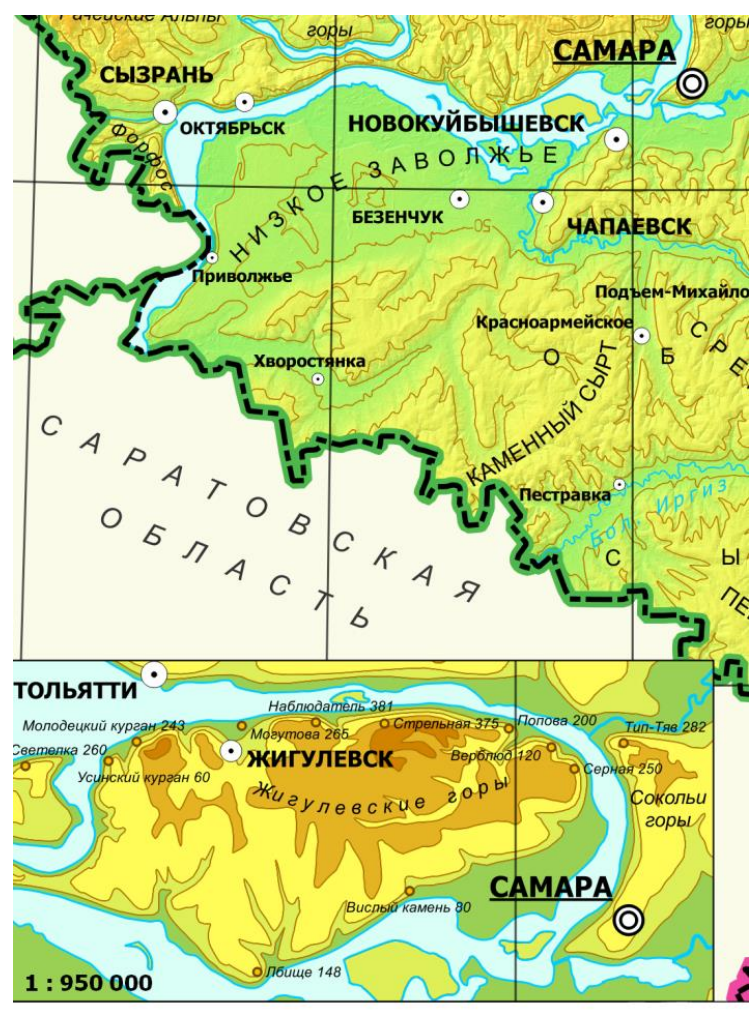

Figure 5. Fragment of the final relief map of Samara Region (Russian Federation)

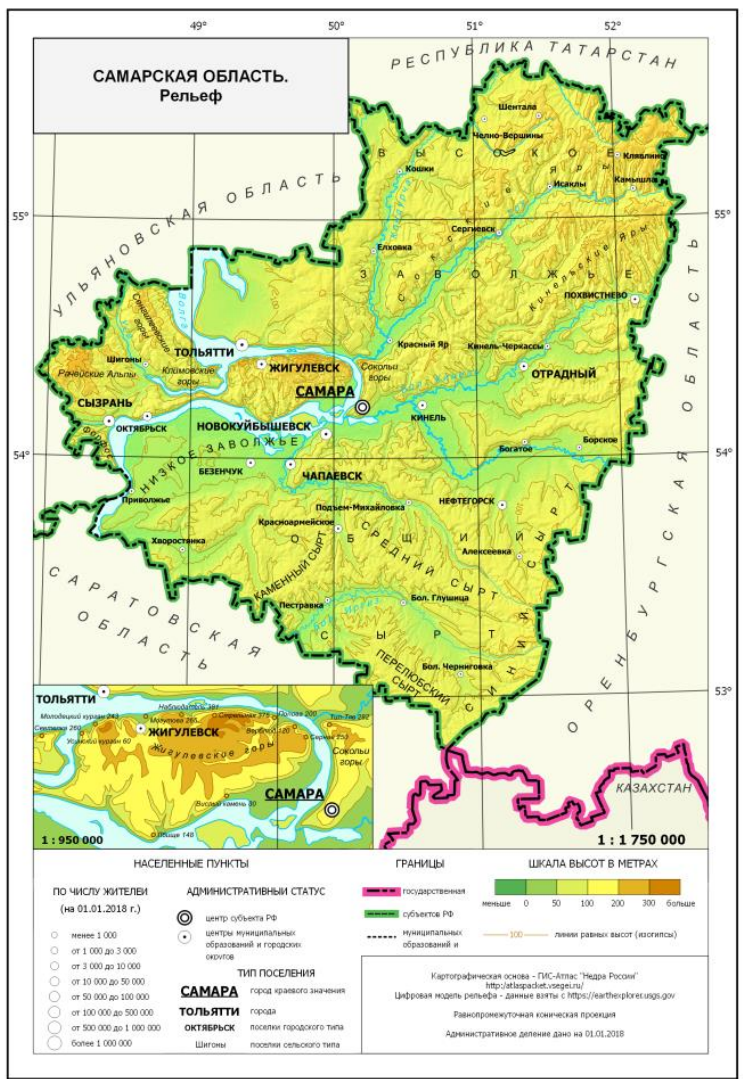

Figure 6. Final relief map of Samara Region (Russian Federation) 
imagery with radiometric calibration and further atmospheric correction can be used to construct a vegetation maps. (Schovengerdt, R., 2010), (Shunlin, 2018)

We suggest using the following principles:

- select images for the period of maximum vegetation (as a rule, midsummer), if hardwood predominate; in the case of the predominance of coniferous forests, we can choose the winter months;

- select images on a date as close as possible (further processing is simplified in this case).

The second stage is pretreatment. If there are several images and the dates of their receipt differ significantly (by more than 1-2 weeks), then it is necessary to perform a radiometric calibration with further atmospheric correction. (Schovengerdt, R., 2010)

This fact is not critical, but if the images have no atmospheric haze or it is sufficiently pure and transparent. During the performing of atmospheric correction, all values will eventually be reduced to the interval [0...1] (with small deviations). Therefore, if such procedures are not performed, you can simply scale the images using a raster calculator:

$$
1 /(\max -\min ) *(\text { rast-min }),
$$

max - the maximum value of raster, min - minimum value, rast value of the current pixel. (Shapiro, M., 2016)

We suggest not to work with superfluous data, but to crop each image along the border of its territory. If the image is cloudy, so it is necessary to put a mask in order to remove it from the further classification, using QA Band. It stores bit pixel values, encoding clouds, shadows from them, snow, etc. The areas shaded in gray indicate the bits that are currently filled in BQA. (Figure 7)

If it is necessary to select regions with the presence of clouds and shadows in them with a probability of at least $33 \%$, then we can use the following expression:

$(($ BQA \& 64) != 0) $\|(($ BQA \& 256) != 0), (Richard, r., 2000)

\begin{tabular}{|c|c|c|c|c|c|c|c|c|c|c|}
\hline \multicolumn{11}{|c|}{ andsat $8 \mathrm{OL}, \mathrm{O}$} \\
\hline BIT & \begin{tabular}{l|l}
15 & 14 \\
\end{tabular} & \begin{tabular}{l|l}
13 & 12 \\
\end{tabular} & \begin{tabular}{l|l}
11 & 10 \\
\end{tabular} & 9 & 7 & 5 & 3 & 2 & 1 & 0 \\
\hline 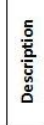 & 总 & 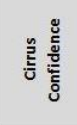 & 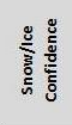 & 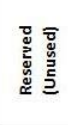 & 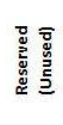 & 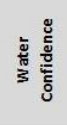 & 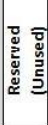 & 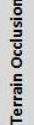 & 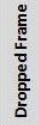 & 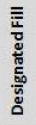 \\
\hline
\end{tabular}

Figure 7. BQA channel coding

If it is necessary to select regions with the presence of clouds and shadows in them with a probability of at least $33 \%$, then you can use the following expression:

$(($ BQA \& 64)! = 0) $\|(($ BQA \& 256)! = 0), (Richard, r., 2000)

BQA is the BQA channel.

The result is a mask, which can then be inverted (using a logical NOT) to select "clean" areas and multiply to other channels to get the same channels that are not affected by clouds and shadows.
The next step - the creation of mosaics. For convenience, all the pieces of scenes obtained from the result of the previous step can be glued together into one image. We can use one of the following options:

- "Raster - Other - Association";

- "Raster - Other - Create a virtual raster (catalog)";

- i.image.mosaic (GRASS command);

- mosaic raster layers (SAGA command)

For better classification, channels: B1, B2, B3, B4, B5, B6 may be suitable. Therefore, it is enough to build mosaics using only bands. (Figure 8)

The next step - the creation of composites. We came to the conclusion that during the work with composites for classification, it is best to combine either B5, B4, B3, or B5, B6, B4. Both combinations are images in pseudo colors. (Quinn, J., 2001) (Figure 9)

One of the last steps is classification. The task of interpreting the classifications of plant communities is one of the most difficult. There are no unique solutions, so we experimented.

It is possible to carry out the classification «supervised learning", it is possible "unsupervised learning". (Lillesand, et al, 2015), (Phiri, et al., 2017). If a classification is "supervised learning", we need to make the training set for each class (forest, water, field, etc.), guided by the following:

- the sample size for each class should be approximately the same: unbalanced data degrade the quality of the model;

- we must try not to mix pixels of different classes: it also directly affects the final result;

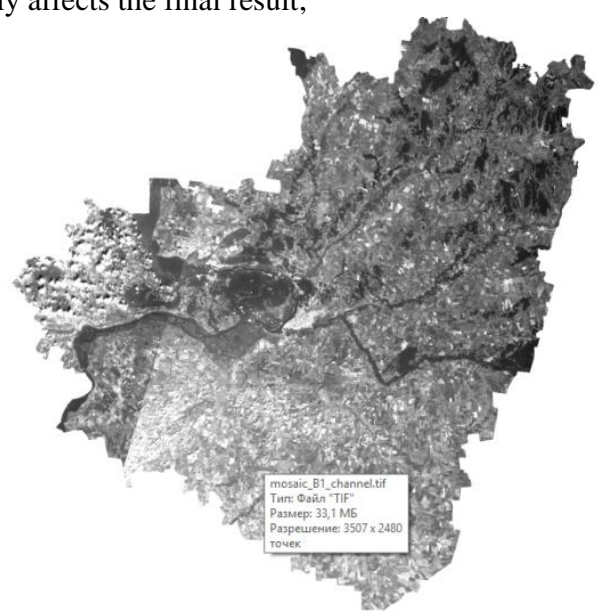

Figure 8. Mosaic_B1_channel. Samara Region (Russian Federation)

- it is necessary to cover as many variations of sign changes for each class.

If preference is given to "unsupervised learning" classification, the number of classes must be allocated intuitively. In this case, if the same object (open ground, field, arable land) has a very wide range of values (from "white" to "black"), it is better to divide this class into subclasses and add their number to the total number of other classes. After classification, they can be combined if necessary. This is because a simple classification is 
based on the type of work with statistical indicators, which are calculated in the process separately for each class. If, for example, the soil has a value from 15 to 200 , and the forest from 100 to 115 , then the average values of both classes will coincide, and an indication will be already useless.

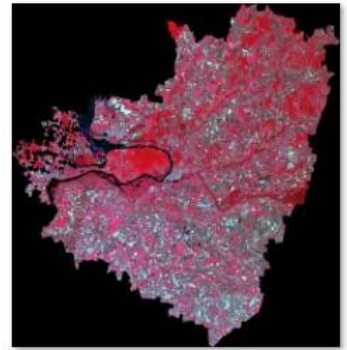

mosaic_B543.tif

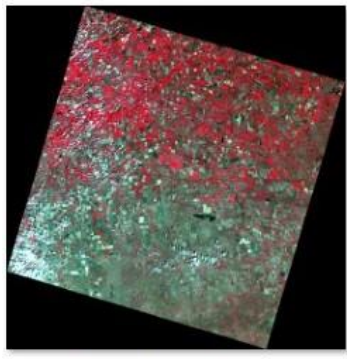

Path_169_Row_24_B543.tif

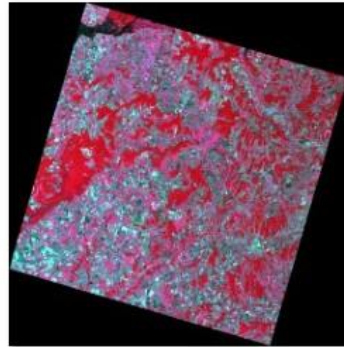

Path_169_Row_22_B543.tif

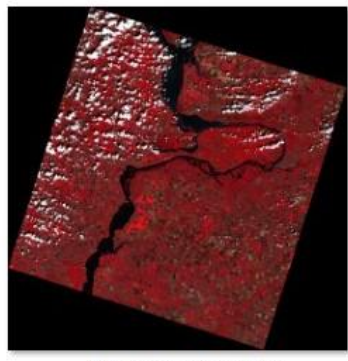

Path_170_Row_23_B543.tif
Figure 9. Construction of mosaics and composites for vegetation maps.

It is possible to use the NDVI with setting thresholds for each class. (Bhandari et al., 2012), (Rouse et al., 1973).

For large-sized areas, we offer the following algorithm:

1. Using of "unsupervised classification": to set the number of classes (10-12) and highlight hydrography. Next, we need to analyze which groups belong to the same class, and combine them.

2. Also, use the "unsupervised classification": take some of the images obtained in the previous step, and containing vegetation. Since forests and some of the fields are similar to each other, they may be in the same class. For the separation of "forest" and "not forests" should once again use "unsupervised classification" (enough to take 5-6 classes and combine them).

3. Use forest vegetation, selected in the previous step, and reuse "unsupervised classification" also, but with 3 classes (mixed coniferous and deciduous forests).

4. Use B1 channel to isolate urban areas use. There may be used as a "supervised learning" and "unsupervised" (simple binary classification of "settlement" - "everything else").

The next step is image post-processing. Before converting the received raster images to vector format, it is possible to try to eliminate the noise (individual pixels or group of pixels). Without this step, the vector may contain thousands of objects, and it will be very massive. To filter the noise, we suggest using the following algorithm: r.neighbors- morphological filter (if the group was small, it will completely disappear) - majority / minority.
To the final vector image we need to apply geographical generalization techniques and coordination with relief. (Figure 10, 11)

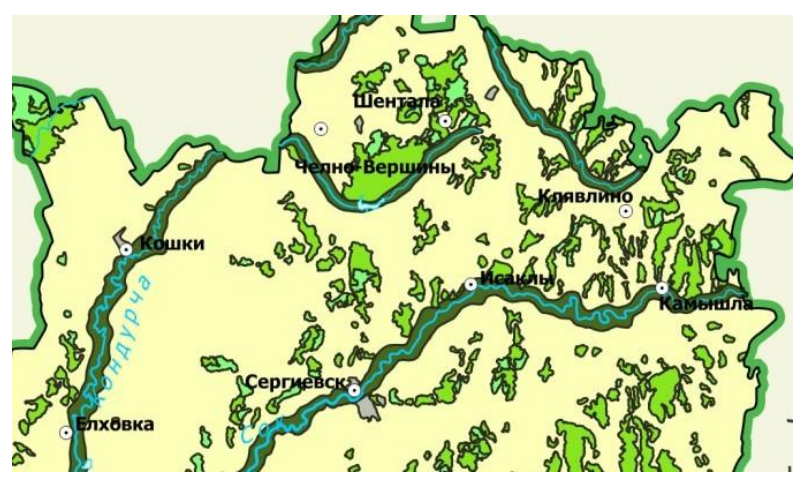

Figure 10. Fragment of the final vegetation map of Samara Region (Russian Federation)

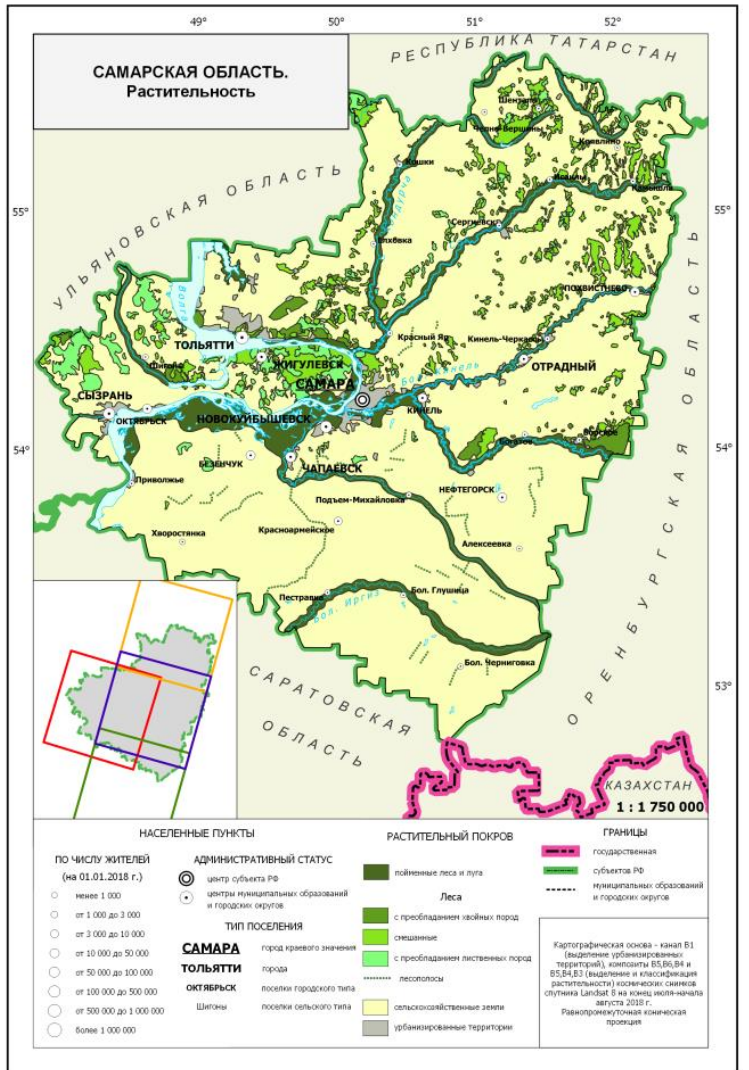

Figure 11. Final vegetation map of Samara Region (Russian Federation)

For any algorithm ENVI (Environment for Visualizing Images) software is most convenient for using. (Harris Geospatial Solutions, Inc., 2019)

\section{RESULTS}

As the result of the work, we propose several algorithms for compiling relief and vegetation maps using remote sensing data: "supervised learning", "unsupervised learning" and via the NDVI index. In addition to these algorithms, we also offer the 
"multi-level supervised learning" algorithm, which can be used for large areas. These are the administrative territories dominated by the Russian Federation and other large countries.

\section{CONCLUSIONS}

As a result of the evaluation of the vegetation and relief maps compilation for large legal administrative territories, we came to the following conclusion:

1. The algorithm of the vegetation and relief maps compilation should contain field reference sites. Photographic interpretation of field reference sites can only be performed by a narrow specialist of the specified profile: botanist, landscape scientist, physical geographer. Decoding the elements of vegetation is also very difficult without the presence of a specialist.

2. The differences between the elements of vegetation are possible to see with the correct combination of satellite images channels only. For a specific area, a specific time of year, a specific task, it is necessary to choose a certain combination of channels. It takes a lot of scientific time.

3. In the Russian Federation all the administrative districts cover large areas. Not all the images on these areas may be necessary quality. But the using of multi-time data is impractical. For large areas, the number of satellite images increases. So the volume and number of errors increases also.

4. In that case if is not possible to pick up a simultaneous imaging of the same spectral range; we have to work with images of different range. Then it is necessary to consider further algorithms for compiling a final map.

5. Often, a large administrative region has large differences in relief and vegetation. This applies particularly to the mountainous areas. In this case, it is advisable to divide the region into parts and continue to work with individual parts, using the capabilities of the algorithms for working with images and computer graphics features to improve visual perception.

6. The problem of small objects generalization requires attention also. It is not always advisable to carry out such algorithms by the method of classical cartography. Small objects of nature can be crucial: oases in the desert, hydrological objects with a stock of fresh water, etc. Automated methods (filtering) should be applied with extreme caution. By this, it is necessary to add a decrease in the resolution of the image.

7. An important problem is the availability of relevant materials on plant communities (vegetation maps) for comparison with reference polygons. Similar thematic editions are not updated every year. In national atlases, information may be too generalized. Special maps may be out of reach. This item requires prior preparation.

8. Processing large volumes of raster data requires powerful and fast computers. Similar types of work are not always carried out in companies that own such technology. The purpose of our work is to show the methodology of working with remote sensing data for the administrations of the regions and large municipalities. A study of the Earth from space is not a priority for the executive authority of the regions. They may not have the necessary high-speed equipment.
9. We think that for the three classical algorithms "supervised learning", "unsupervised learning" and via the NDVI index, we have to add the algorithm "multi-level supervised learning". We define this method as the primary one.

10. The main goal of this study is the choice of the most optimal way to compiling visual models of vegetation and relief for a large administrative territory using remote sensing data.

\section{REFERENCES}

Bhandari, A., Kumar, R., Singh, G., 2012; Feature Extraction using Normalized Difference Vegetation Index (NDVI): a Case Study of Jabalpur City; Elsevier Ltd. Selection and peer-review under responsibility of the Department of Computer Science \& Engineering, National Institute of Technology Rourkela DOI: 10.1016/j.protcy.2012.10.074)

Bozilina, A., Emeljanova, L., Kotova, T., at. Al., 2016. Geographical mapping: maps of nature: study guide, Moscow, Publishing house KDU, Russia, P. 316 (in Russian)

Harris Geospatial Solutions, Inc, 2019, ENVI, Software and Technology, harrisgeospatial.com/SoftwareTechnology/ENVI, (20 February, 2019)

Isachenko, A., 1960, Physical-geographical mapping, Part 2.3. L: Publishing house of Leningrad State University, P. 231(in Russian)

Kennelly, P., 2008, Terrain maps displaying hill-shading with curvature; Geomorphology 102 (2008) 567-577; DOI: 10.1016/j.geomorph.2008.05.046

Lillesand, T., Kiefer, R.. Chipman, J., Remote sensing and image interpretation, ISBN: 978-1-118-34328-9- 7th Ed. $2015,736 \mathrm{P}$

Metelka, V., et al., 2018, Automated regolith landform mapping using airborne geophysics and. remote sensing data, Burkina Faso, West Africa, DOI: 10.1016/j.rse.2017.05.004

Phiri, D., Morgenroth, J., 2017, Developments in Landsat Land Cover Classification Methods; Remote sensing: Review; DOI: $10.3390 /$ rs 9090967

Quinn, J., 2001, Summary of Band Combinations, web.pdx.edu/ nauna/resources/10_BandCombinations February 2019)

Richard R., Irish Landsat 7 Automatic Cloud Cover Assessment Article in Proceedings of SPIE - The International Society for Optical Engineering 4049 . August 2000, DOI: $10.1117 / 12.410358$

Rouse, J., Haas, R., Shell, J., Deering, D., 1973, Monitoring vegetation systems in the Great Plains with ERTS. In 3rd ERTS Symposium, NASA SP-351 I, P. 309-317

Schovengerdt, R., 2010, Remote sensing. Models and methods of image processing, Moscow: Technosphere, 2010. - $556 \mathrm{P}$., ISBN 978-5-94836-244-1, FB 2 10-52/11

Shunlin, L., et al., 2018, Atmospheric Correction of Landsat ETM+ Land Surface Imagery-Part I: Methods; DOI: 10.1109/36.964986) 
Seger, M, 1989, Remote-sensing cartography with satellite photography- digital methods, relief map-making, examples of geographical science, Mittelungen der osterreichischen geographischen gesellschaft, Vienne, Austria, WOS: A1989CT36300028, P.311-312

Shapiro, M., U.S.Army Construction Engineering Research Laboratory Glynn Clements Last changed: \$Date: 2016-06-01 16:10:45 -0700 (Wed, 01 Jun 2016)

Shuttle Radar Topography Mission, 2018, Srtm.csi.cgiar.org/srtmdata (1 February 2019)

Sochava, V., 1979, Vegetation cover on thematic maps, Novosibirsk: Science, 1979, P. 189 (in Russian)

USGS, United States Geological Survey (USGS), 2018a, Data and Tools. earthexplorer.usgs.gov (1 February 2019).

USGS, United States Geological Survey (USGS), 2018b, Landsat Collections, doi.org/10.3133/fs20183049

Zaruzkaja, I., Krasilnikova, N., Design and mapping: maps of nature, 1989, Moscow State University, Russia, P. 296 (in Russian) 